\title{
GC-MS Analysis of Bioactive Phytochemicals in Methanol Extract of Aerial Part and Callus of Dipterygium glaucum Decne
}

\author{
Choudhary D, Shekhawat JK, Kataria V*
}

\section{Choudhary D, Shekhawat JK, Kataria V*}

Biotechnology Unit, Department of Botany (UGC-Centre of Advanced Study), Jai Narain Vyas University, New Campus, Jodhpur 342001, Rajasthan, INDIA.

\section{Correspondence}

\section{Dr. Vinod Kataria}

Assistant Professor, Biotechnology Unit, Department of Botany (UGC-CAS), Jai Narain Vyas University, New Campus, Jodhpur 342001, Rajasthan, INDIA.

Phone no: +912912722555,+919772287375

E-mail: vinodkataria2002@gmail.com, vk.bo@jnvu.edu.in

History

- Submission Date: 10-07-2019.

- Review completed: 24-07-2019;

- Accepted Date: 07-08-2019.

DOI : 10.5530/pj.2019.11.165

Article Available online

http://www.phcogj.com/v11/i5

\section{Copyright}

(c) 2019 Phcogi.Com. This is an openaccess article distributed under the terms of the Creative Commons Attribution 4.0 International license.

\begin{abstract}
Introduction: This study was designed to evaluate the phytocomponents present in aerial part and in vitro induced callus methanol extracts of Dipterygium glaucum by Gas Chromatography-Mass Spectrometry (GC-MS) technique. Methods: GC-MS analysis of aerial part sample and callus produced from leaf of $D$. glaucum extracted in methanol solvents was performed using GC-MS QP 2010 Plus (Shimadzu, Japan) system comprising an auto sampler (AOC-20i) and a gas chromatograph interfaced to a mass spectrometer. Results: This study was carried out to identified and comparative analysis of bioactive phytochemicals from aerial part extract and callus extract of $D$. glaucum. This analysis revealed that both the extracts have 69 different types of phytochemical components in varying quantities. Some of the important phytochemical compounds were Stigmasterol, $\beta$-sitosterol, $\gamma$-sitosterol, Campesterol, Squalene, n-Hexadecanoic acid, Stearic acid, Myristic acid, Quinazoline, Linalyl acetate etc. These chemical compounds have anticancer, antitumor, anti-inflammatory, antidiabetic and antioxidants properties. Conclusion: This study represents the detection and identification of different phytochemical compounds from aerial part and callus extract of $D$. glaucum. Thus, due to the presence of various important bioactive phytocomponents this plant is recommended as a pharmaceutically important plant.
\end{abstract}

Key words: Callus, Campesterol, Capparidaceae, Fatty acid, Stigmasterol, Terpenoids.

\section{INTRODUCTION}

Plants are an important component of the health care system and are being used as a source of medicine due to their therapeutic potential since ancient times. The presence of the phytochemicals such as alkaloids, flavonoids and phenolic compounds in medicinal plants have been part of phytomedicine. ${ }^{1}$ Phytochemicals are natural bioactive plant derived chemicals which play a vital role in the treatment and prevention of several diseases. The potential efficacy and safety of many plant-based drugs have already been proven. ${ }^{2}$ The phytochemicals in the plant extract target the biochemical pathway, therefore the traditional drugs are safer than synthetic drugs. ${ }^{3}$ Now-a-days, synthetic medicines are widely used which may cause severe side effects in body and these consequences are sometimes more serious than that of disease itself. Therefore, to overcome this situation, pharmaceutical companies are spending a lot of time and money on the medicinal plant extracts for the formulation of natural drugs which are safe and cost effective i.e., affordable for common people. ${ }^{4}$ World Health Organization (WHO) in 2013, launched 'WHO Traditional Medicine Strategy 2014-2023' which emphasized to promote universal healthcare by joining traditional and complementary medicines together and to ensure the quality, effectiveness and safety of such medicines. ${ }^{5}$ Plants are rich source of secondary metabolites that have diverse biological activities and these metabolites serve as active medicines against several diseases. ${ }^{4,6}$ Nearly $20 \%$ of plant species have been examined in both biological or pharmacological applications to confirm their benefits and safety. ${ }^{7}$ In the recent times, a wide attention has been given to the studies on phytoconstituents of medicinal plants and its pharmacological activities. ${ }^{8,9}$ Various solvent system like water, ethanol, methanol, chloroform and ethyl acetate have been used for the extraction of secondary metabolites, such as steroids, terpenes, alkaloids, tannins and phenols. ${ }^{4}$ Many advanced techniques are adapted for the identification and quantification of bioactive compounds in plant materials and one of them, is Gas ChromatographyMass Spectrometry (GC-MS) technique. GCMS technique has become a key of technological platform for profiling of secondary metabolite ${ }^{10}$ and direct analysis of unknown components existing in medicinal plants. ${ }^{11}$

A monotypic genus with one species, Dipterygium glaucum Decne., locally known as "Phel" belongs to the family Capparidaceae is distributed across the N.E. Africa, Arabia, Sind and India. ${ }^{12}$ In India it is distributed on gravely grounds and sand dunes in the desert areas of NW Rajasthan and Gujarat. ${ }^{13} D$. glaucum is an important medicinal perennial shrub and used to cure respiratory diseases, skin diseases, wounds and chronic fever..$^{14}$ This plant is a source of various phytochemicals such as volatile alkaloids, cyanides, coumarins and flavonoids ${ }^{15}$ with multiple biological activities like insecticidal, anthelmintic, antileishmanial, antibacterial and antifungal. ${ }^{16}$ The aerial plant parts of D. glaucum is used in bronchial asthma as bronchodilator ${ }^{17}$ while the whole plant is used as decoction and infusion for jaundice, psoriasis

Cite this article: Choudhary D, Shekhawat JK, Kataria V. GC-MS Analysis of Bioactive Phytochemicals in Methanol Extract of Aerial Part and Callus of Dipterygium glaucum Decne. Pharmacog J. 2019;11(5):1055-63. 
and ring worms. ${ }^{12}$ D. glaucum have significant antioxidant activities and shows cytotoxic activity against cervical and breast cancer carcinoma cells. $^{18}$ Thus, D. glaucum have enormous therapeutic potential and these properties demand phytochemical analysis, evaluation and validation for natural product development.

The present study is focused to evaluate and analyse the presence of phytoconstituents in methanolic extracts of aerial part and in vitro grown callus of Dipterygium glaucum in order to identify and characterize bioactive compounds with their concentration through a GC-MS technique.

\section{MATERIALS AND METHODS}

\section{Instrument and chemicals}

Gas Chromatograph-Mass Spectrometer system (GCMS QP 2010, Shimadzu, Japan), Shaker, Borosil beaker $(500 \mathrm{ml}$, Borosil Glass works Ltd., Mumbai, India), Methanol (Analytical grade,), Murashige and Skoog (MS) medium PT-100 (Murashige and Skoog 1962), 6-Benzyladenine (BA; Sigma-Aldrich) and 2,4-dichlorophenoxyacetic acid (2,4-D; Sigma-Aldrich $\left.{ }^{\circledR}\right)$.

\section{Plant material and callus culture}

The fresh aerial parts of Dipterygium glaucum were collected in the month of February from Jaisalmer, an arid region of the Thar desert of Rajasthan, India (Figure 1). The aerial parts were rinsed thoroughly with autoclaved distil water to remove dust particles and dried at room temperature under the shade for nearly 20 days. The dried plant parts were pulverized to fine powder using mechanical grinder and kept in air tight polybags till further use. To analyse the callus culture of $D$. glaucum, in vitro callus culture has been established from leaf explant. The desired amount of callus was produced on MS medium augmented $0.5 \mathrm{mgl}^{-1}$ each BA and 2,4-D (Figure 2).

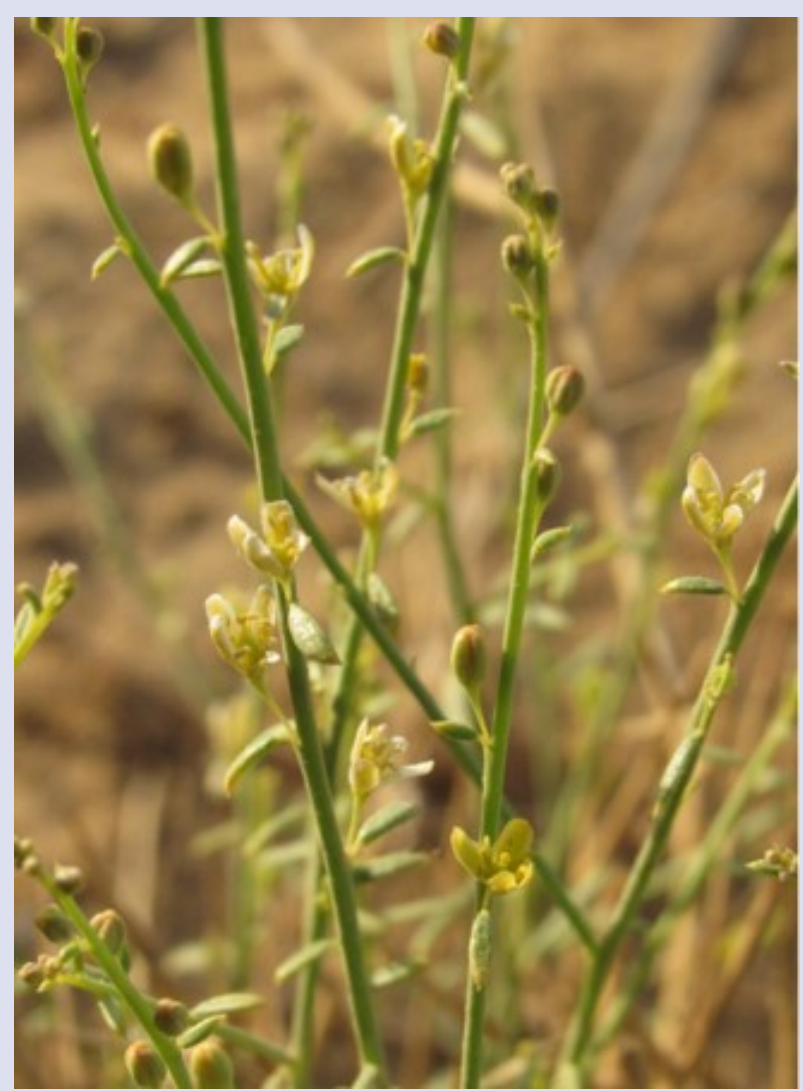

Figure 1: Dipterygium glaucum in its natural habitat.

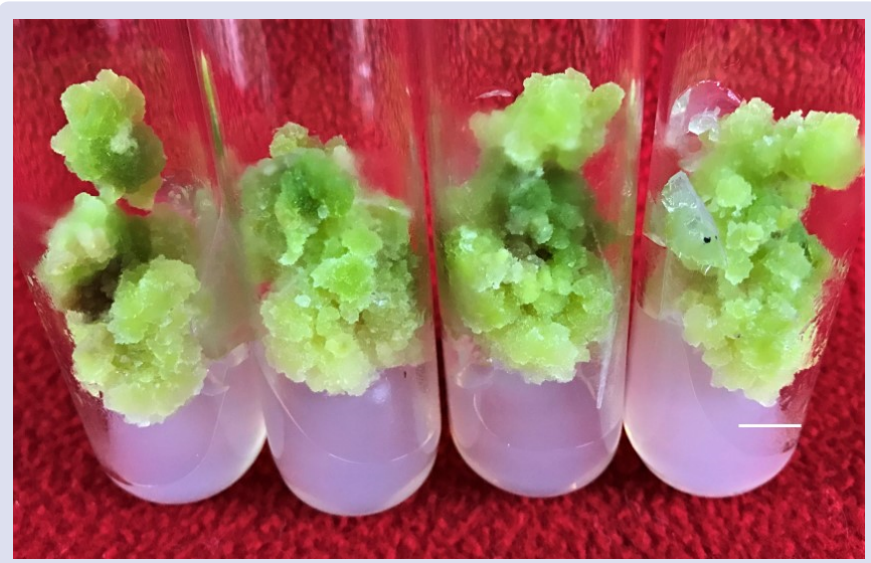

Figure 2: In vitro callus culture establishment from leaf of Dipterygium glaucum on MS medium augmented with $0.5 \mathrm{mg} \mathrm{l}^{-1}$ each of BA and 2,4D. BA: 6-Benzyladenine and 2,4-D: 2,4-dichlorophenoxyacetic acid. (Scale bar $=10 \mathrm{~mm}$ )

\section{Preparation of extracts}

The powdered samples (aerial part and callus) of D. glaucum were extracted with methanol at room temperature. $30 \mathrm{~g}$ of aerial part powder and $10 \mathrm{~g}$ of callus were weighed, treated with $500 \mathrm{ml}$ of methanol in a beaker covered with aluminium foil and kept on shaker for 2-3 days with occasional stirring. The extracts were filtered through muslin cloth followed by Whatman filter paper No. 1. and to obtain clear solution, the filtrate was further centrifuged at $2500 \mathrm{rpm}$ for 10 minutes. The clear filtrate was evaporated to dryness to obtain the final concentrated extract and that extract was analysed by using GC-MS.

\section{Gas chromatography-mass spectrometry (GC-MS) analysis}

For GC-MS analysis, $1 \mu \mathrm{l}$ of stock solution sample $(1 \mathrm{mg} / \mathrm{ml}$ crude extract in methanol) was used. GC-MS analysis was performed at USIC, AIRF, Jawaharlal Nehru University, New-Delhi with GC-MS QP 2010 Plus (Shimadzu, Kyoto, Japan) system comprising an auto sampler (AOC-20i) and gas chromatograph interfaced to a mass spectrometer with an ion source temperature $\left(220^{\circ} \mathrm{C}\right)$ and interface temperature $\left(270^{\circ} \mathrm{C}\right)$. For GC-MS detection, a threshold desorption system was operated with an ionization energy of $1000 \mathrm{ev}$ and mass range of $50-650 \mathrm{~m} / \mathrm{z}$ purposed. The column oven temperature was $60^{\circ} \mathrm{C}$ and injection temperature $260^{\circ} \mathrm{C}$ at a pressure of $93.1 \mathrm{kPa}$. The carrier gas used was helium (99.99\%) as a constant flow rate-total flow: 19.5 $\mathrm{ml} / \mathrm{min}$ and column flow: $1.50 \mathrm{ml} / \mathrm{min}$. The GC-MS program total running time was 50 minutes. The software used for the analysis of mass spectra and chromatograms was a Turbo mass and the relative percentage of each component was calculated by comparing its average peak area to the total area.

\section{Identification of phytocomponents}

The phytochemical components extracted were identified by comparing the retention time and molecular weight of the detected components with the mass spectra of the known components available in the Willey libraries and National Institute of Standards and technology (NIST) library.

\section{RESULTS}

The GC-MS chromatograms spectra of the methanolic extract of aerial part and callus revealed the richness of phytochemical compounds in Dipterygium glaucum and contribute to the medicinal quality of the plant (Figures 3 and 4). The GC-MS analysis of aerial part extract and 


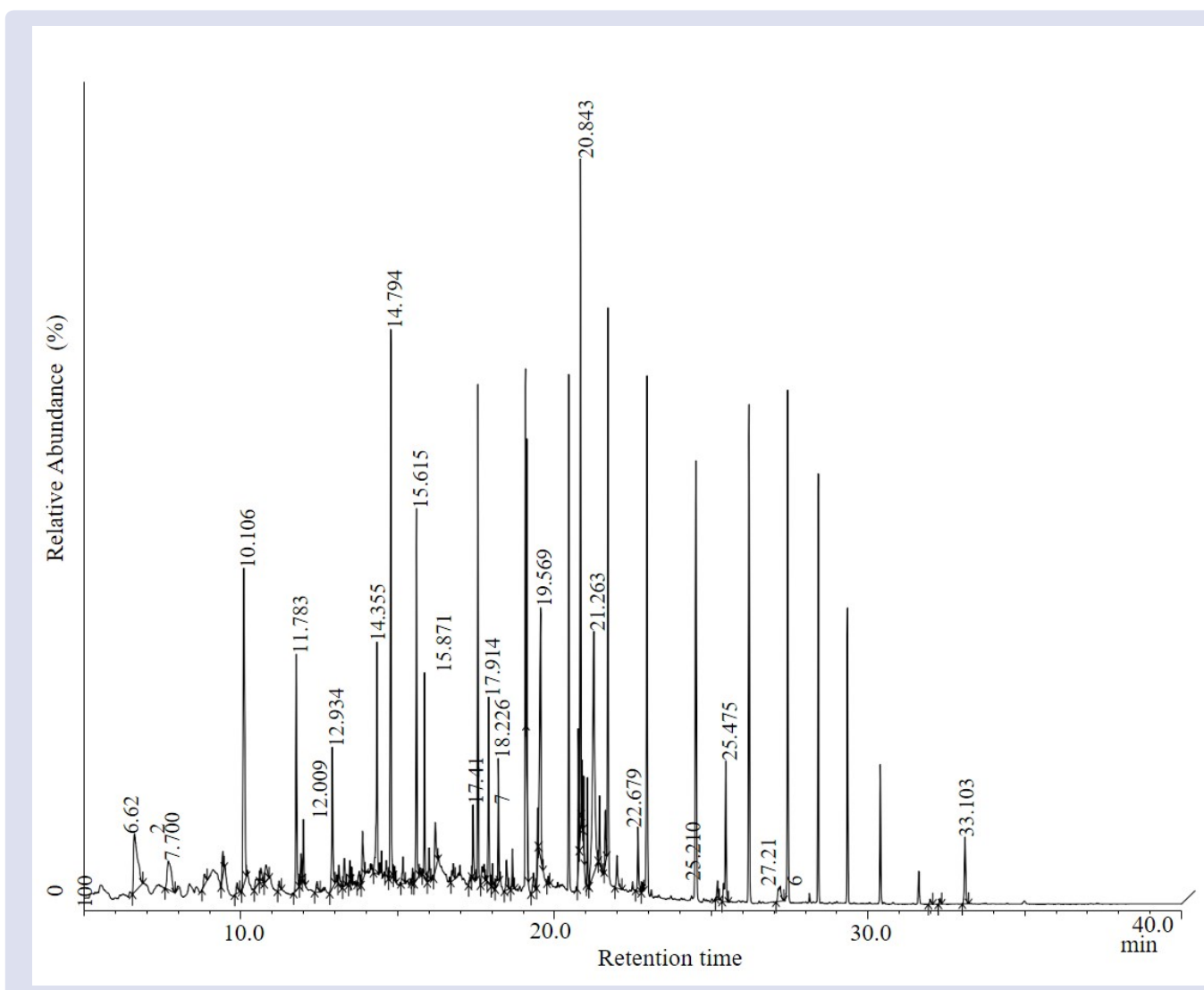

Figure 3: GC-MS chromatogram of methanolic extract of aerial part showing relative abundance and retention time of Dipterygium glaucum.

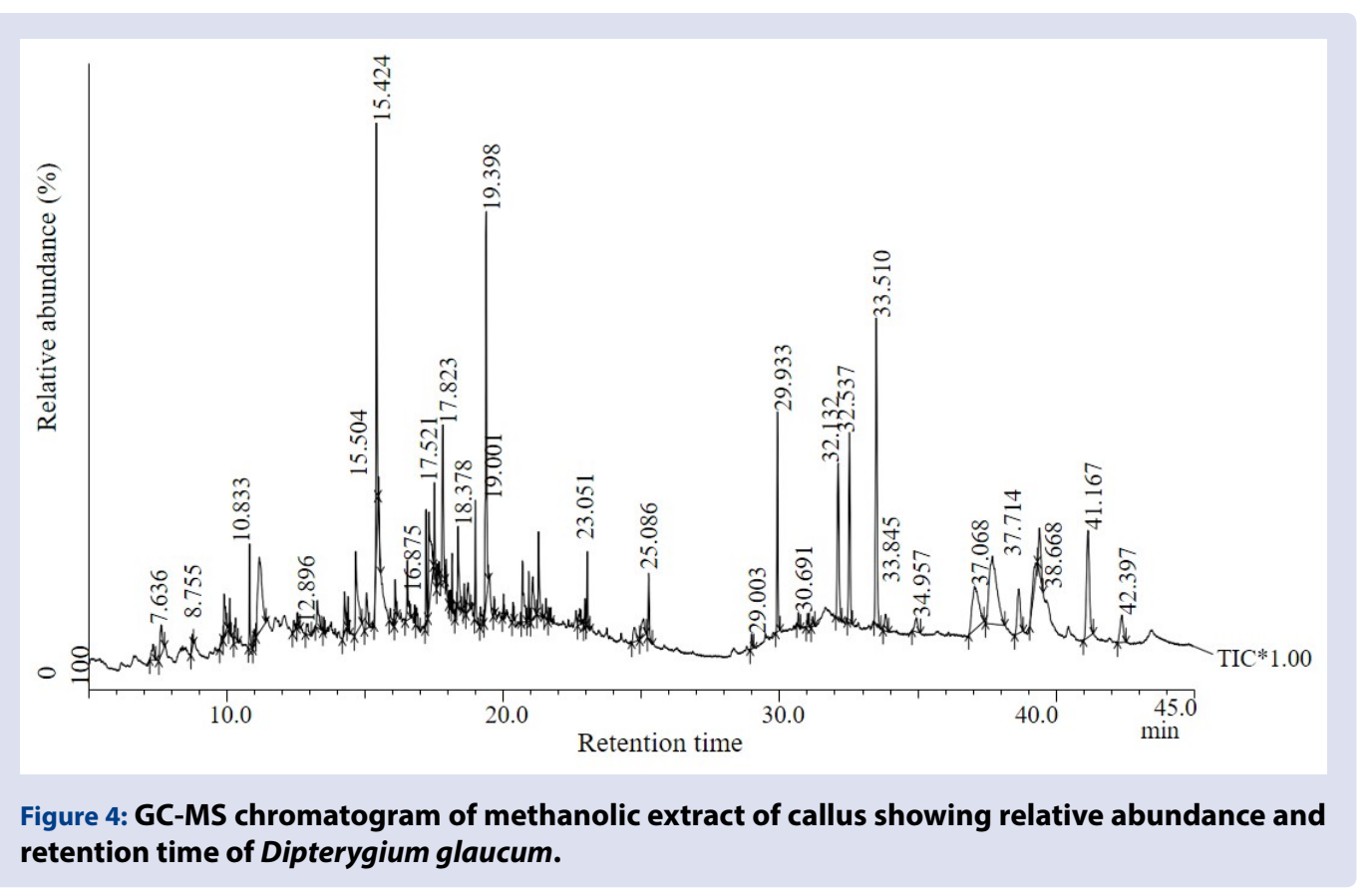

callus extract indicated the presence of 33 and 36 bioactive compounds respectively. The name and nature of the identified phytochemical compounds in aerial part and callus with their retention time, molecular formula, molecular weight and abundance (peak area in \%) are presented in Tables 1 and 2 respectively. The major compounds in aerial part extract were- 9,12-Octadecadienoyl chloride, $(\mathrm{Z}, \mathrm{Z})-(8.86 \%)$,
Dibenzofuran (8.40\%), 9-Octadecenoic acid (Z)-, methyl ester (8.18\%), Azulene (8.17\%), n-Hexadecanoic acid (5.54), Fluorene (5.36\%), Acenaphthylene (4.07\%) Benzenesulfonic acid, 4-Hydroxy (3.65\%), $\beta$-Sitosterol (1.43\%) and Phytol (0.68\%). The callus cells also expressed good amount of phytoconstituents which was proved by the GCMS analysis of the callus produced from leaf explant of Dipterygium 
Table 1: List of compounds identified from different classes of bioactive phytochemicals by GC-MS of methanolic extract of aerial part of Dipterygium glaucum.

\begin{tabular}{|c|c|c|c|c|c|c|}
\hline S.No. & Compound name & $\begin{array}{l}\text { Molecular } \\
\text { Formula }\end{array}$ & $\begin{array}{l}\text { Molecular } \\
\text { weight } \\
\left(\mathrm{g} \mathrm{mol}^{-1}\right)\end{array}$ & $\begin{array}{l}\text { Retention time } \\
\text { (min) }\end{array}$ & $\begin{array}{l}\text { Peak Area } \\
\text { (\%) }\end{array}$ & Compound type \\
\hline 1. & Benzenesulfonic acid, 4-Hydroxy & $\mathrm{C}_{6} \mathrm{H}_{6} \mathrm{O}_{4} \mathrm{~S}$ & 174 & 6.62 & 3.65 & Phenolic \\
\hline 2. & $\begin{array}{l}\text { 4H-Pyran-4-one, } \\
\text { 3-Hydroxy-2-methyl }\end{array}$ & $\mathrm{C}_{6} \mathrm{H}_{6} \mathrm{O}_{3}$ & 126 & 8.85 & 0.39 & Organic compound \\
\hline 3. & 1,5-Anhydro-6-deoxyhexo-2,3-diulose & $\mathrm{C}_{6} \mathrm{H}_{8} \mathrm{O}_{4}$ & 144 & 9.44 & 0.56 & Glycoside \\
\hline 4. & Phenol, 2,4-dichloro- & $\mathrm{C}_{6} \mathrm{H}_{4} \mathrm{CL}_{2} \mathrm{O}$ & 162 & 9.89 & 0.30 & Phenolic \\
\hline 5. & Azulene & $\mathrm{C}_{10} \mathrm{H}_{8}$ & 128 & 10.10 & 8.17 & Hydrocarbon \\
\hline 6. & 1,2-Benzenediol & $\mathrm{C}_{6} \mathrm{H}_{6} \mathrm{O}_{2}$ & 110 & 10.48 & 0.37 & Phenolic \\
\hline 7. & 2,3-Dihydro-benzofuran & $\mathrm{C}_{8} \mathrm{H}_{8} \mathrm{O}$ & 120 & 10.65 & 0.43 & Phenolic \\
\hline 8. & 5-Hydroxymethylfurfural & $\mathrm{C}_{6} \mathrm{H}_{6} \mathrm{O}_{3}$ & 126 & 10.81 & 0.62 & $\begin{array}{l}\text { Benzyl or furfuryl } \\
\text { alcohol }\end{array}$ \\
\hline 9. & Naphthalene, 1-methyl- & $\mathrm{C}_{11} \mathrm{H}_{10}$ & 142 & 11.78 & 4.00 & Hydrocarbon \\
\hline 10. & 2-Methoxy-4-vinylphenol & $\mathrm{C}_{9} \mathrm{H}_{10} \mathrm{O}_{2}$ & 150 & 11.93 & 0.44 & Phenolic \\
\hline 11. & $\begin{array}{l}\text { 1,7,7-Trimethyl bicyclo[2.2.1]heptan-2- } \\
\text { one }\end{array}$ & $\mathrm{C}_{10} \mathrm{H}_{14} \mathrm{D}_{2} \mathrm{O}$ & 154 & 12.44 & 0.33 & Terpene \\
\hline 12. & Naphthalene, 2,6-dimethyl- & $\mathrm{C}_{12} \mathrm{H}_{12}$ & 156 & 13.31 & 0.97 & Hydrocarbon \\
\hline 13. & Acenaphthylene & $\mathrm{C}_{12} \mathrm{H}_{10}$ & 154 & 14.35 & 4.07 & Hydrocarbon \\
\hline 14. & Dibenzofuran & $\mathrm{C}_{12} \mathrm{H}_{8} \mathrm{O}$ & 168 & 14.79 & 8.40 & Phenol \\
\hline 15. & 1,2-Benzenedicarboxylic acid & $\mathrm{C}_{24} \mathrm{H}_{38} \mathrm{O}_{4}$ & 390 & 15.49 & 0.21 & Phenol \\
\hline 16. & Fluorene & $\mathrm{C}_{13} \mathrm{H}_{10}$ & 166 & 15.61 & 5.36 & Hydrocarbon \\
\hline 17. & Phenanthrene & $\mathrm{C}_{14} \mathrm{H}_{10}$ & 178 & 17.91 & 2.75 & Hydrocarbon \\
\hline 18. & Neophytadiene & $\mathrm{C}_{20} \mathrm{H}_{38}$ & 278 & 18.22 & 1.67 & Terpenoid \\
\hline 19. & Tridecanal & $\mathrm{C}_{13} \mathrm{H}_{26} \mathrm{O}$ & 198 & 18.48 & 0.62 & - \\
\hline 20. & 3,7,11,15-Tetramethyl-2-hexadecen-1-ol & $\mathrm{C}_{20} \mathrm{H}_{40} \mathrm{O}$ & 296 & 18.67 & 0.54 & Terpene \\
\hline 21. & Hexadecanoic acid, methyl ester & $\mathrm{C}_{17} \mathrm{H}_{34} \mathrm{O}_{2}$ & 270 & 19.13 & 2.85 & Fatty acid ester \\
\hline 23. & n-Hexadecanoic acid & $\mathrm{C}_{16} \mathrm{H}_{32} \mathrm{O}_{2}$ & 256 & 19.56 & 5.54 & Fatty acid \\
\hline 24. & 9,12-Octadecadienoic acid, methyl ester & $\mathrm{C}_{19} \mathrm{H}_{34} \mathrm{O}_{2}$ & 294 & 20.77 & 2.42 & Fatty acid ester \\
\hline 25. & 9-Octadecenoic acid (Z)-, methyl ester & $\mathrm{C}_{19} \mathrm{H}_{36} \mathrm{O}_{2}$ & 296 & 20.84 & 8.18 & Fatty acid ester \\
\hline 26. & Phytol & $\mathrm{C}_{20} \mathrm{H}_{40} \mathrm{O}$ & 296 & 20.93 & 0.68 & Terpene \\
\hline 27. & Octadecanoic acid, methyl ester & $\mathrm{C}_{19} \mathrm{H}_{38} \mathrm{O}_{2}$ & 298 & 21.06 & 1.30 & Fatty acid ester \\
\hline 28. & 9,12-Octadecadienoyl chloride, $(\mathrm{Z}, \mathrm{Z})$ - & $\mathrm{C}_{18} \mathrm{H}_{31} \mathrm{ClO}$ & 298 & 21.26 & 8.86 & Fatty acid \\
\hline 29. & 9-Octadecenoic acid (Z)- & $\mathrm{C}_{18} \mathrm{H}_{34} \mathrm{O}_{2}$ & 282 & 21.44 & 1.12 & Fatty acid \\
\hline 30. & $\begin{array}{l}\text { Hexadecanoic acid, 2-hydroxy-1- } \\
\text { (hydroxymethyl)ethyl ester }\end{array}$ & $\mathrm{C}_{19} \mathrm{H}_{38} \mathrm{O}_{4}$ & 330 & 25.21 & 0.36 & Fatty acid ester \\
\hline 31. & Di-n-octyl phthalate & $\mathrm{C}_{24} \mathrm{H}_{38} \mathrm{O}_{4}$ & 390 & 25.47 & 2.60 & Ester \\
\hline 32. & Ethyl iso-allocholate & $\mathrm{C}_{26} \mathrm{H}_{44} \mathrm{O}_{5}$ & 436 & 31.99 & 0.11 & Alkaloid \\
\hline 33. & $\beta$-Sitosterol & $\mathrm{C}_{29} \mathrm{H}_{50} \mathrm{O}$ & 414 & 33.10 & 1.43 & Steroid \\
\hline
\end{tabular}

glaucum. The major compounds of callus extract were-, Stigmasterol (7.51\%), Cholesta-3,5-dien-7-one (6.42\%), n-Hexadecanoic acid (6.47\%), Ergost-5-en-3-ol, (3.beta., 24R)- (4.18\%), Cholesta-4,6-Dien3-ol, Benzoate, (3.beta.)- (3.95\%), Squalene (3.17\%), Phenanthrene (3.14\%), Tetradecanoic acid (3.07\%), Octadecanoic acid (1.50\%), $\gamma$-sitosterol (1.20\%). The components found similar in both the extracts were 1,2 Benzenedicarboxylic acid, n-Hexadecanoic acid, Phenanthrene, Hexadecanoic acid, Methyl ester and Fluorene.

\section{DISCUSSION}

Gas chromatography coupled with mass spectrometry (GC-MS) is preferred for more precise information in both qualitative analysis and for quantitative determination. ${ }^{19}$ The D. glaucum plant was found to be rich in steroids, terpenes and terpenoids, fatty acid and their esters, hydrocarbons, phenolic and alcoholic compounds. Present study shows the medicinal values of $D$. glaucum by identifying various phytochemical compounds through GC-MS analysis of aerial part and callus. The bioactive phytochemical constituents reported in $D$. glaucum through the present study are from group of Terpenes and Terpenoids (Eucalyptol; Neophytadiene; 3,7,11,15-Tetramethyl-2- hexadecen-1-ol / Phytol; Linalyl acetate; Alpha-guaiene, etc.), Steroids ( $\beta$-sitosterol; $\gamma$-sitosterol; Stigmasterol; Ergost-5-en-3-ol, (3.beta., 24R)/ Campesterol; Squalene; etc.), Fatty acids and esters (n-Hexadecanoic acid; Hexadecanoic acid, methyl ester; 9-Octadecanoic acid, methyl ester; Tetradecanoic acid; Oleic acid; etc.), Phenolic compounds (1,2-Benzenediol; 2-Methoxy-4-vinylphenol; 2,3-Dihydro-benzofuran; etc.), Alkaloids (Quinazoline) and Hydrocarbons (Azulene; etc.). The biological activities and uses of some important phytochemical constituents are summarized in Table 3.

\section{Steroids}

Overall, the richness of steroids is more in callus extract than the aerial part of D. glaucum. Various steroids were present in callus extract such as Squalene, Stigmasterol, Campesterol, $\gamma$ - sitosterol. Similar to our observation, presence of good amount of steroid in callus culture has been reported by Galane ${ }^{20}$ in Solanum aviculare. The Squalene compound has antitumor, antioxidant, anticancer, antimicrobial, chemo-preventive, pesticide and sun-screen properties. ${ }^{21}$ Squalene has also been reported as an important precursor for the synthesis of phytosterols such as Sitosterol, Campesterol and Stigmasterol. ${ }^{22}$ 
Table 2: List of compounds identified from different classes of bioactive phytochemicals by GC-MS of methanolic extract of callus of Dipterygium glaucum.

\begin{tabular}{|c|c|c|c|c|c|c|}
\hline S.No. & Compound name & Molecular Formula & $\begin{array}{l}\text { Molecular } \\
\text { weight } \\
\left(\mathrm{g} \mathrm{mol}^{-1}\right)\end{array}$ & $\begin{array}{l}\text { Retention time } \\
\text { (min) }\end{array}$ & $\begin{array}{l}\text { Peak Area } \\
(\%)\end{array}$ & Compound type \\
\hline 1. & Eucalyptol & $\mathrm{C}_{10} \mathrm{H}_{18} \mathrm{O}$ & 154 & 7.35 & 0.56 & Terpene \\
\hline 2. & 2-Butanone,3-methyl-1-phenyl- & $\mathrm{C}_{11} \mathrm{H}_{14} \mathrm{O}$ & 162 & 7.63 & 1.02 & Phenolic \\
\hline 3. & Linalyl acetate & $\mathrm{C}_{12} \mathrm{H}_{20} \mathrm{O}_{2}$ & 196 & 10.83 & 1.30 & Terpenes \\
\hline 4. & Quinazoline & $\mathrm{C}_{8} \mathrm{H}_{6} \mathrm{~N}_{2}$ & 130 & 11.00 & 0.29 & Alkaloids \\
\hline 5. & Z,Z,Z-1,4,6,9-Nonadecatetraene & $\mathrm{C}_{19} \mathrm{H}_{32}$ & 260 & 12.43 & 0.24 & Alkene \\
\hline 6. & n-Decanoic acid & $\mathrm{C}_{10} \mathrm{H}_{20} \mathrm{O}_{2}$ & 172 & 12.57 & 0.26 & Fatty acid \\
\hline 7. & $\begin{array}{l}\text { 1,3-Isobenzofurandione, 3a,4,7,7a-tetrahydro- } \\
\text { 5-methyl- }\end{array}$ & $\mathrm{C}_{9} \mathrm{H}_{10} \mathrm{O}_{3}$ & 166 & 13.28 & 0.76 & Esters \\
\hline 8. & Alpha-guaiene & $\mathrm{C}_{15} \mathrm{H}_{24}$ & 204 & 13.51 & 0.16 & Sesquiterpenes \\
\hline 9. & Phenol,3,5-bis(1,1-dimethylethyl)- & $\mathrm{C}_{14} \mathrm{H}_{22} \mathrm{O}$ & 206 & 14.39 & 0.42 & Phenolic \\
\hline 10. & Fluorene & $\mathrm{C}_{13} \mathrm{H}_{10}$ & 166 & 15.50 & 0.28 & Hydrocarbon \\
\hline 11. & $\begin{array}{l}\text { 1,6-methanonaphthalen } 1(2 \mathrm{H}) \text {-ol, octahydro } \\
\text { 4,8A,9,9-tetramethyl-, (1R,4s,4as,6R,8as) }\end{array}$ & $\mathrm{C}_{15} \mathrm{H}_{26} \mathrm{O}$ & 222 & 16.54 & 1.16 & Sesquiterpenes \\
\hline 12. & Tetradecanoic acid & $\mathrm{C}_{14} \mathrm{H}_{28} \mathrm{O}_{2}$ & 228 & 17.32 & 3.07 & Fatty acid \\
\hline 13. & Benzyl Benzoate & $\mathrm{C}_{14} \mathrm{H}_{12} \mathrm{O}_{2}$ & 212 & 17.52 & 1.32 & Esters \\
\hline 14. & Phenanthrene & $\mathrm{C}_{14} \mathrm{H}_{10}$ & 178 & 17.82 & 3.14 & Hydrocarbon \\
\hline 15. & Isopropyl myristate & $\mathrm{C}_{17} \mathrm{H}_{34} \mathrm{O}_{2}$ & 270 & 17.94 & 0.35 & Esters \\
\hline 16. & 2-Pentadecanone,6,10,14-trimethyl & $\mathrm{C}_{18} \mathrm{H}_{36} \mathrm{O}_{2}$ & 268 & 18.15 & 0.53 & Diterpenoids \\
\hline 17. & 1-Octadecanol & $\mathrm{C}_{18} \mathrm{H}_{38} \mathrm{O}$ & 270 & 18.73 & 0.86 & Fatty alcohol \\
\hline 18. & Hexadecanoic acid, methyl ester & $\mathrm{C}_{17} \mathrm{H}_{34} \mathrm{O}_{2}$ & 270 & 19.00 & 1.43 & Fatty acid ester \\
\hline 19. & Palmitoleic acid & $\mathrm{C}_{16} \mathrm{H}_{30} \mathrm{O}_{2}$ & 254 & 19.19 & 0.43 & Fatty acid \\
\hline 20. & n- Hexadecanoic acid & $\mathrm{C}_{16} \mathrm{H}_{32} \mathrm{O}_{2}$ & 256 & 19.39 & 6.47 & Fatty acid \\
\hline 21. & Ethylene brassylate & $\mathrm{C}_{15} \mathrm{H}_{26} \mathrm{O}_{4}$ & 270 & 20.02 & 0.28 & Ester \\
\hline 22. & Heptadecanoic acid & $\mathrm{C}_{17} \mathrm{H}_{34} \mathrm{O}_{2}$ & 270 & 20.36 & 0.24 & Fatty acid \\
\hline 23. & 9-Octadecanoic acid, methyl ester & $\mathrm{C}_{19} \mathrm{H}_{34} \mathrm{O}_{2}$ & 296 & 20.70 & 1.49 & Fatty acid ester \\
\hline 24. & Oleic acid & $\mathrm{C}_{18} \mathrm{H}_{34} \mathrm{O}_{2}$ & 282 & 21.07 & 1.28 & Fatty acid \\
\hline 25. & Octadecanoic acid & $\mathrm{C}_{18} \mathrm{H}_{36} \mathrm{O}_{2}$ & 284 & 21.29 & 1.50 & Stearic acid \\
\hline 27. & 4,8,12,16-Tetramethylheptadecan-4-olide & $\mathrm{C}_{21} \mathrm{H}_{40} \mathrm{O}_{2}$ & 324 & 23.05 & 0.99 & Terpenes \\
\hline 28. & 1,2 Benzenedicarboxylic acid & $\mathrm{C}_{24} \mathrm{H}_{38} \mathrm{O}_{4}$ & 390 & 25.27 & 1.09 & Phenolic \\
\hline 29. & Squalene & $\mathrm{C}_{30} \mathrm{H}_{50}$ & 410 & 29.93 & 3.17 & Steroid \\
\hline 30. & Tetrapentacontane & $\mathrm{C}_{54} \mathrm{H}_{110}$ & 759 & 31.02 & 0.22 & - \\
\hline 31. & Cholesta-4,6-Dien-3-ol, Benzoate, (3.beta.)- & $\mathrm{C}_{34} \mathrm{H}_{48} \mathrm{O}_{2}$ & 488 & 32.13 & 3.95 & Steroid \\
\hline 32. & Ergost-5-en-3-ol, (3.beta., 24R)- & $\mathrm{C}_{28} \mathrm{H}_{48} \mathrm{O}$ & 400 & 37.06 & 4.18 & Steroid \\
\hline 33. & Stigmasterol & $\mathrm{C}_{29} \mathrm{H}_{48} \mathrm{O}$ & 412 & 37.71 & 7.51 & Steroid \\
\hline 34. & $\gamma$-Sitosterol & $\mathrm{C}_{29} \mathrm{H}_{50} \mathrm{O}$ & 414 & 39.26 & 1.20 & Steroid \\
\hline 35. & Cholesta-3,5-dien-7-one & $\mathrm{C}_{27} \mathrm{H}_{42} \mathrm{O}$ & 382 & 41.16 & 6.42 & Steroid \\
\hline 36. & Stigmast-4-en-3-one & $\mathrm{C}_{29} \mathrm{H}_{48} \mathrm{O}$ & 412 & 42.39 & 1.23 & Steroid \\
\hline
\end{tabular}

Phytosterols have antitumoral, anticancerous, anti-inflammatory, antiatherogenic, anti-ulcerative, antifungal and antibacterial activities. ${ }^{23-25}$ In addition, Stigmasterol (7.51\%), a major compound present in callus extract, is an unsaturated phytosterol with multiple medicinal properties like anti-osteoarthritic, cholesterol lowering, ${ }^{26}$ thyroid inhibitory, hypoglycemic, antiperoxidative, ${ }^{27}$ anti-inflammatory, antiviral, antihepatotoxic, cancer preventive activities, ${ }^{28}$ antioxidant and it also shows antibacterial activity against multidrug resistant mycobacterium. ${ }^{29}$ Similarly, Cheong et al..$^{22}$ reported the presence of Stigmasterol in the callus extract of Strobilanthes crispus. Ergost-5-en3-ol,(3.beta., 24R)- (4.18\%) also known as Campesterol is a cholesterol absorption reducing agent and possess antioxidant and anticancerous activities. ${ }^{30}$ The presence of $\gamma$-sitosterol or Clionasterol was detected in callus extract of D. glaucum and shows anti-cancerous, ${ }^{31}$ hepatoprotective, antihyperglycemic activity and act as an antidiabetic drug. ${ }^{32,33} \beta$-sitosterol (1.43\%) was the only steroid present in the aerial part extract and shows different biological activities like antitumor, ${ }^{34}$ antiproliferative, ${ }^{35}$ antioxidative, antiviral, immunomodulatory and hepatoprotective activity. ${ }^{36}$ Some other steroidal compounds present in callus extract were Cholesta-3,5-dien-7-one (6.42\%), Cholesta-4,6- dien-3-ol, Benzoate, (3.beta., 24R)- (3.95\%) and Stigmast-4-en-3-one (1.23\%).

\section{Terpenes and Terpenoids}

Terpenes and terpenoidshas important physiological as well as ecological roles and shows many biological activities such as hepato-protective, antioxidant and cholinesterase inhibitors. ${ }^{11}$ Total ten different terpenes and terpenoids compounds were present in the extracts of D. glaucum. Neophytadiene (1.67\%), a terpenoid compound, was found in the aerial part extract and has anti-inflammatory, antipyretic, antioxidant and antimicrobial properties. ${ }^{37}$ It is also used in treatment of rheumatism, headache and skin problems. ${ }^{38} 3,7,11,15$ - Tetramethyl-2-hexadecen-1ol $(0.54 \%)$, commonly known as Phytol, a monounsaturated diterpene alcohol present in aerial part extract. This compound known to possess anticancer, anti-inflammatory, antidiuretic, antimicrobial, ${ }^{29}$ antioxidant, immunostimulant and cholesterol lowering properties. ${ }^{11}$ Correspondingly, Ogunlesi et al..$^{39}$ and Satyal et al..$^{40}$ identified Phytol in leaves of Euphorbia hirta and Cassia fistula, respectively, which act as a precursor of Vitamins (E \& K), including the cytotoxic activity against 
Table 3: Some important phytochemical constituents identified in methanolic extracts of aerial part and callus of $D$. glaucum and their biological activities.

\begin{tabular}{|c|c|c|c|c|}
\hline S.No. & Compound name & Compound class & $\begin{array}{l}\text { Aerial } \\
\text { part/ } \\
\text { Callus }\end{array}$ & Uses / Biological activities \\
\hline 1. & $\beta$-Sitosterol & Steroid & $\mathrm{Ae}$ & $\begin{array}{l}\text { Antitumor, }{ }^{34} \text { antiproliferative, }{ }^{35} \text { antioxidative, immunomodulatory, } \\
\text { antiviral and hepatoprotective }{ }^{36}\end{array}$ \\
\hline 2. & $\gamma$-Sitosterol & Steroid & $\mathrm{Ca}$ & $\begin{array}{l}\text { Anti-cancerous }{ }^{31}, \text { antihyperglycemic activity, hepatoprotective and } \\
\text { antidiabetic drug } \\
\text { 32-33 }\end{array}$ \\
\hline 3. & Stigmasterol & Steroid & $\mathrm{Ca}$ & $\begin{array}{l}\text { Anti-osteoarthritic }{ }^{26} \text {, antiperoxidative, thyroid inhibitory }{ }^{27} \text {, } \\
\text { antihepatotoxic, anti-inflammatory, antiviral, cancer- preventive }{ }^{28}\end{array}$ \\
\hline 4. & $\begin{array}{l}\text { Ergost-5-en-3-ol, (3.beta., } \\
\text { 24R)- }\end{array}$ & Steroid & $\mathrm{Ca}$ & Antioxidant, anti-cancerous, hypocholesterolemic ${ }^{30}$ \\
\hline 5. & Eucalyptol & Terpenes & $\mathrm{Ca}$ & Anti-inflammatory ${ }^{43}$ \\
\hline 6. & 1,2-Benzenediol & Phenolic & $\mathrm{Ae}$ & Antioxidant, anti-cancer and pesticides ${ }^{54}$ \\
\hline 7. & Neophytadiene & Terpenoid & Ae & Antipyretic, antioxidant, antimicrobial and anti-inflammatory ${ }^{37}$ \\
\hline 8. & $\begin{array}{l}\text { 3,7,11,15-Tetramethyl-2- } \\
\text { hexadecen-1-ol }\end{array}$ & Terpene & $\mathrm{Ae}$ & Antimicrobial, anti-inflammatory, anti-cancer and anti-diuretic ${ }^{29}$ \\
\hline 9. & n-Hexadecanoic acid & Fatty acid & $\mathrm{Ae} / \mathrm{Ca}$ & $\begin{array}{l}\text { Anti-cancerous }{ }^{47} \text {, anti-inflammatory }{ }^{48} \text {, antioxidant, anti-androgenic, } \\
\text { hypo-cholesterolemic, hemolytic, } 5 \text { - alpha reductase inhibitor, } \\
\text { nematicides and pesticides }{ }^{19}\end{array}$ \\
\hline 10. & 2-Methoxy-4-vinylphenol & Phenolic & $\mathrm{Ae}$ & Anti-inflammatory, anti-microbial, analgesic and antioxidant ${ }^{51}$ \\
\hline 11. & 2,3-Dihydro-benzofuran & Phenolic & Ae & Diabetic retinopathy and arthritis ${ }^{38}$ \\
\hline 12. & $\begin{array}{l}\text { Hexadecanoic acid, methyl } \\
\text { ester }\end{array}$ & Fatty acid ester & $\mathrm{Ae} / \mathrm{Ca}$ & $\begin{array}{l}\text { Antitumor, immunostimulant }{ }^{11} \text {, antioxidant, } 5 \text {-alpha reductase } \\
\text { inhibitor, hypo-cholesterolemic and pesticides }{ }^{19}\end{array}$ \\
\hline 13. & Linalyl acetate & Terpene & $\mathrm{Ca}$ & Anti-inflammatory ${ }^{41}$ \\
\hline 14. & Quinazoline & Alkaloids & $\mathrm{Ca}$ & Antitumor ${ }^{65}$ \\
\hline 15. & $\begin{array}{l}\text { 9-Octadecenoic acid, methyl } \\
\text { ester }\end{array}$ & Fatty acid ester & $\mathrm{Ae}$ & $\begin{array}{l}\text { Antiandrogenic, cancer preventive, anti-inflammatory, anemiagenic, } \\
\text { dermatitigenic and insectiguge }^{10}\end{array}$ \\
\hline 16. & Ethyl Iso-Allocholate & Alkaloid & Ae & Anti-inflammatory and anti-microbial agents ${ }^{64}$ \\
\hline 17. & Tetradecanoic acid & Fatty acid & $\mathrm{Ca}$ & Cancer preventive, antioxidant, ${ }^{49}$ antifungal and antibacterial ${ }^{50}$ \\
\hline 18. & Oleic acid & Fatty acid & $\mathrm{Ca} / \mathrm{Ae}$ & $\begin{array}{l}\text { Anti-inflammatory, cancer preventive, anti-androgenic, anemiagenic, } \\
\text { 5-alpha reductase inhibitor, dermatitigenic, hypocholesterolemic, } \\
\text { insectifuge }^{51}\end{array}$ \\
\hline 19. & Alpha-guaiene & Sesquiterpene & $\mathrm{Ca}$ & Anti-cancerous $^{44}$ \\
\hline 20. & Azulene & Hydrocarbon & $\mathrm{Ae}$ & Anti-inflammatory ${ }^{62}$ \\
\hline 21. & Squalene & Steroid & $\mathrm{Ca}$ & $\begin{array}{l}\text { Antioxidant, anticancerous, antitumor, antimicrobial, } \\
\text { chemopreventive and pesticides }{ }^{21}\end{array}$ \\
\hline
\end{tabular}

Ae: Aerial part; Ca: Callus.

breast cancer cell line. Linalyl acetate (1.30\%), a monoterpenoid and 1,6-methanonaphthalen 1(2H)-ol,octahydro 4,8A,9,9-tetramethyl,(1R,4s,4as,6R,8as) (Patchouli alcohol) (1.16\%), a tricyclic sesquiterpene were present in the callus extract and shows anti-inflammatory activity. ${ }^{41,42}$ Eucalyptol (1.8 -cineol) (0.56\%), saturated monoterpene, a major constituent of eucalyptus oil shows anti-inflammatory activity and also used in remedy of sinusitis, bronchitis and colds. ${ }^{43}$ Alphaguaiene $(0.16 \%)$, a constituent of Agarwood, a sesquiterpenoids was also present in callus extract and shows anti-cancerous activity. ${ }^{44}$ The 4,8,12,16- tetramethylheptadecan-4-olide (0.99\%), an isoprenoid $\gamma$-lactone is also found in callus extract.

\section{Fatty acids and their esters}

Presence of fatty acids and their esters were in abundance in both the extracts of D. glaucum and 18 major fatty acids compounds were found. Fatty acids show antibacterial activity and widely used in drug preparations, fat emulsions, cosmetics and liposomes. ${ }^{45,46}$ Palmitic acid or n-Hexadecanoic acid, a saturated fatty acid was found in both, aerial part $(5.54 \%)$ and callus extract (6.47\%). It has anti-cancerous, ${ }^{47}$ antiinflammatory, ${ }^{48}$ antioxidant, anti-androgenic, hypocholesterolemic, 5-alpha reductase inhibitor, nematicides and insecticides properties. ${ }^{19}$ In addition, another compound Hexadecanoic acid, methyl ester was also present in both aerial part extract $(2.85 \%)$ and callus extract
(1.43\%). It is a saturated fatty acid ester and shows antitumor, immunostimulant ${ }^{11}$, antioxidant, 5 -alpha reductase inhibitor, pesticides and hypocholesterolemic properties. ${ }^{19}$ Stearic acid and Myristic acid are saturated fatty acids and found in callus extract. Myristic acid or Tetradecanoic acid (3.07\%) has cancer preventive, antioxidant, ${ }^{49}$ antifungal and antibacterial properties..$^{50}$ Stearic acid or Octadecanoic acid (1.50\%) shows antimicrobial activity. ${ }^{50}$ Oleic acid or 9-Octadecenoic acid, a mono unsaturated fatty acid present in aerial part extract $(1.12 \%)$ and callus extract (1.28\%). It has anti-inflammatory, cancer preventive, anti-androgenic, 5-alpha reductase inhibitor, anemiagenic, insectifuge, dermatitigenic and hypocholesterolemic properties. ${ }^{51}$ Moreover, it is also used in aerosol products as an emulsifying or solubilizing agent. ${ }^{52}$ Another saturated fatty acid found in the callus extract was Heptadecanoic acid (0.24\%). It has anti-inflammatory, antibacterial, antimicrobial, hypocholesterolemic and cancer preventive activities. ${ }^{29}$ In aerial part extract, a linoleic acid ester group compounds were identified, namely, 9- Octadecenoic acid (Z)- methyl ester (8.18\%) and 9,12- Octadecadienoic acid, methyl ester (2.42\%) which have anti-androgenic, anti-inflammatory, hypocholesterolemic, and cancer preventive properties. ${ }^{10}$ The presence of another compound Hexadecanoic acid, 2-hydroxy-1-(hydroxymethyl) ethyl ester (0.36\%) in aerial part which has medicinal properties such as anti-oxidant, anthelmintic and anti-inflammatory ${ }^{53}$ Overall abundant fatty acids were present in D. glaucum. 


\section{Alcoholic and phenolic compounds}

The aerial part extract has 8 alcoholic and phenolic compounds whereas 5 were present in callus extract. The phenolic compound, 1,2Benzenedicarboxylic acid, also known as Phthalic acid, was found in both aerial part (0.21\%) and callus extract (1.09\%). Phenolic compounds present in the aerial part extract were 2-Methoxy-4-vinylphenol (0.44\%), 2,3- Dihydro-benzofuran (0.43\%) and 1,2- Benzenediol (0.37\%). The phenolic compound, 2-Methoxy-4-vinylphenol possess antiinflammatory, antimicrobial and antioxidant activity. ${ }^{51}$ 2,3- Dihydrobenzofuran or Coumaran an essential oil which is used to cure arthritis and diabetic retinopathy. ${ }^{38}$ The presence of another important phenolic compound was Catechol or 1,2- Benzenediol which shows anticancer, analgesic and antioxidant properties. ${ }^{54} \mathrm{It}$ is also used as a raw material for drugs, pesticides, polymerization inhibitors, dyes, leather tanning, analytical reagents and deoxygenating agent. ${ }^{55} 2$-Butanone,3-methyl-1phenyl (1.02\%), 1-Octadecanol (0.86\%) and 10-Nonadecanol (0.35\%) compounds were present in the callus extract. 10-Nonadecanol, an alcoholic compound possesses nematicides and pesticides properties. ${ }^{56}$

\section{Esters}

Besides fatty acid esters, a total of 5 ester compounds were also detected. Among the detected esters, the Benzyl benzoate (1.32\%), a natural compound present in the callus extracts shows anti-hypertensive effects by suppressing the Ang-II induced hypertension. ${ }^{57}$ Isopropyl myristate $(0.35 \%)$, an ester of isopropyl alcohol and myristic acid and ethylene brassylate were used in cosmetics, perfumery ${ }^{58}$ and pharmaceuticals ointments. ${ }^{59}$

\section{Hydrocarbons}

In the extracts of D. glaucum, total 8 different hydrocarbons were detected. Among them Fluorene and Phenanthrene were present in both the aerial part and callus extracts. Fluorene, an aromatic hydrocarbon having medicinal values, used in production of drugs and in pharmaceuticals ${ }^{60}$ whereas, Phenanthrene, a natural compound has been reported for its anticancer, anti-inflammatory, antimicrobial, spasmolytic, phytotoxicity, antiplatelet aggregation and antiallergic activities. ${ }^{61}$ The compound Azulene (8.17\%) was found in the aerial part extract and shows anti-inflammatory activity. ${ }^{62}$

\section{Other compounds}

Some other compounds were found in the aerial part extract of $D$. glaucum such as 1,5-Anhydro-6-deoxyhexo-2,3-diulose (0.56\%), a glycoside which is used as preservative $e^{63}$ and Ethyl iso-allocholate $(0.11 \%)$, an alkaloid showing anti-inflammatory and antimicrobial properties ${ }^{64}$ Another compound of interest found in callus extract was an alkaloid Quinazoline (0.29\%) which shows anti-tumour activity ${ }^{65}$

\section{CONCLUSION}

Dipterygium glaucum is a medicinal plant and represent rich source of phytochemical compounds having various biological activities. The results of GC-MS analysis presented a comparative study of various phytochemical compounds found in methanolic extract of aerial part and in vitro induced callus of D. glaucum. Total 69 bioactive compounds were identified with medicinal value as well as biological activity. Some important bioactive phytocomponents present in $D$. glaucum were Stigmasterol, Campesterol, $\beta$-sitosterol, $\gamma$ - sitosterol, n-Hexadecanoic acid, Tetradecanoic acid, Quinazoline, Squalene, Oleic acid, etc. It could be concluded that besides aerial part, the in vitro produced callus also possesses various bioactive photochemical compounds from different categories of bioactive compound such as terpenes, steroids, fatty acids, esters, hydrocarbons, phenolic, alcoholic, etc. Till now, no work has been carried out to identify the biological potential of this medicinal plant. Hence it is the first report on GC-MS analysis of methanolic extract of this medicinal plant of arid region.

\section{ACKNOWLEDGEMENT}

Authors acknowledge to the University Grant Commission (UGC), New Delhi, for providing Special Assistance Program (SAP) in the form of Centre of Advanced Study (CAS) to the Department of Botany, Jai Narain Vyas University, Jodhpur and also thankful to the AIRF, JNU, New Delhi for GC-MS analysis.

\section{CONFLICT OF INTEREST}

The authors declare that they have no conflict of interest to publish this manuscript.

\section{SUMMARY}

This study presented a comparative analysis of methanolic extract of aerial part and in vitro induced callus of Dipterygium glaucum using Gas Chromatography-Mass Spectrometry analysis. A total of 69 compounds identified belonging to various classes of compounds with different pharmaceutical properties. The outcome of this study presented that the callus extract also express good amount of bioactive compounds. Some of the important phytochemical compounds were Stigmasterol, $\gamma$-sitosterol, $\beta$-sitosterol, Campesterol, Squalene, Stearic acid, n-Hexadecanoic acid, Myristic acid, Quinazoline, Linalyl acetate etc. Dipterygium glaucum can become a good source for therapeutic studies.

\section{REFERENCES}

1. Edeoga HO, Okwu DE, Mbaebie BO. Phytochemical constituents of some Nigerian medicinal plants. Afr J Biotechnol. 2005;4(7):685-8.

2. Sofowora A, Ogunbodede E, Onayade A. The role and place of medicinal plants in the strategies for disease prevention. Afr J Tradit Complement Altern Med. 2013;10(5):210-29.

3. Rajeswari J, Rani S. GC-MS analysis of whole plant of Leptadenia reticulata. Int J Pharm Tech Res. 2014;6(7):2043-50.

4. Gupta D, Kumar M. Evaluation of in vitro antimicrobial potential and GC-MS analysis of Camellia sinensis and Terminalia arjuna. Biotechnol Rep. 2017;13:19-25.

5. World health organization, Geneva. WHO Traditional Medicine Strategy 20142023. Geneva: World Health Organization. 2013.

6. Rungsung W, Ratha KK, Dutta S, Dixit AK, Hazra J. Secondary metabolites of plants in drugs discovery. World J Pharm Res. 2015;4(7):604-13.

7. Altemimi A Lakhssassi N, Baharlouei A Watson DG, Lightfoot DA Phytochemicals: Extraction, isolation and identification of bioactive compounds from plant extracts. Plants. 2017;6(42):1-23.

8. Krishnamoorthy $K$, Krishnaswamy $T$, Subramaniam $P$, Sellamuthu $M$ Quantification of phytochemicals and in vitro antioxidant potential of various solvent extract of certain species of Acanthaceae. Int J Green Pharm. 2014;8(1):58-64.

9. Alese MO, Adewole OS, Ljomone OM, Ajayi SA, Alese OO. Hypoglycemic and hypolipidemic activities of methanolic extract of Sphenocentrum jollyanum on streptozotocin-induced diabetic wister rats. European J Med Plants. 2014;4(3):353-64.

10. Krishnamoorthy K, Subramaniam P. Phytochemical profiling of leaf, stem, and tuber parts of Solena amplexicaulis (Lam.) Gandhi using GC-MS. Int Scholar Res Not. 2014

11. Godara P, Dulara BK, Barwer N, Chaudhary NS. Comparative GC-MS Analysis of Bioactive Phytochemicals from Different Plant Parts and Callus of Leptadenia reticulata Wight and Arn. Pharmacogn J. 2019;11(1):129-40.

12. Ahmad S, Alam K, Wariss HM, Anjum S, Mukhtar M. Ethnobotanical studies of plant resources of Cholistan desert, Pakistan. Int J Sci Res. 2014;3(6):1782-8.

13. Bhandari MM. Flora of the Indian desert. MPS Repros, Jodhpur. 1990.

14. Malik S, Ahmad S, Sadiq A, Alam K, Wariss HM, Ahmad I, et al. A comparative ethno-botanical study of Cholistan (an arid area) and Pothwar (a semi-arid area) of Pakistan for traditional medicines. J Ethnobiol Ethnomed. 2015;11(1):1-20.

15. Moussa SAI, Taia WK, Al-Ghamdy FGM. Acclimation of Dipterygium glaucum Decne grown in the Western Coastal part of Saudi Arabia to different water supplies. Int J Res Chem Environ. 2012;2(1):301-9. 
16. Hameed M, Ashraf M, Al-Quriany F, Nawaz T, Ahmad MSA, Younis A, et al. Medicinal flora of the Cholistan desert: a review. Pak J Bot. 2011;43:39-50

17. Abdel-Mogib M, Ezmirly ST, Basaif SA. Phytochemistry of Dipterygium glaucum and Capparis decidua. J Saudi Chem Soc. 2000;4(1):103-8.

18. Shaheen U, Shoeib NA, Temraz A, Abdelhady MI. Flavonoidal constituents, antioxidant, antimicrobial, and cytotoxic activities of Dipterygium glaucum grown in Kingdom of Saudi Arabia. Pharmacogn Mag. 2017;13:484-8.

19. Sermakkani M, Thangapandian V. GC-MS analysis of Cassia italica leaf methanol extract. Asian J Pharm Clin Res. 2012;5(2):90-4.

20. Galanes IT, Webb DT, Rosario O. Steroid production by callus and cell suspension cultures of Solanum aviculare. J Nat Prod. 1984;47(2):373-6.

21. Ezhilan BP, Neelamegam R. GC-MS analysis of phytocomponents in the ethanol extract of Polygonum chinense L. Phcog Res. 2011;4(1):11-4.

22. Cheong BE, Zakaria NA, Cheng AYF, Teoh PL. GC-MS Analysis of Strobilanthes crispus plants and callus. Transactions on Science and Technology. 2016;3(12):155-61.

23. Othman RA, Moghadasian MH. Beyond cholesterol-lowering effects of plant sterols: clinical and experimental evidence of anti-inflammatory properties. Nutr Rev. 2011;69(7):371-82.

24. Awad AB, Downie A, Fink CS, Kim U. Dietary phytosterol inhibits the growth and metastasis of MDA-MB-231 human breast cancer cells grown in SCID mice. Anticancer Res. 2000;20:821-4.

25. Berger A, Jones PJ, Abumweis SS. Plant sterols: Factors affecting their efficacy and safety as functional food ingredients. Lipids Health Dis. 2004;3(1):1-19.

26. Gabay O, Sanchez C, Salvat C, Chevy F, Breton M, Nourissat G, et al. Stigmasterol: A phytosterol with potential anti-osteoarthritic properties. Osteoarthritis Cartilage. 2010;18(1):106-16.

27. Panda S, Jafri M, Kar A, Meheta BK. Thyroid inhibitory, antiperoxidative and hypoglycemic effects of stigmasterol isolated from Butea monosperma. Fitoterapia. 2009;80(2):123-6.

28. Beckstrom-Sternberg SM, Duke JA. CRC handbook of medicinal mints (aromathematics): phytochemicals and biological activities. CRC press, Inc, USA. 1996;409-17.

29. Mujeeb F, Bajpai P, Pathak N. Phytochemical evaluation, antimicrobial activity and determination of bioactive components from leaves of Aegle marmelos. Biomed Res Int. 2014;497-606.

30. U.S. Department of agriculture, Agricultural research service. 1992-2016. Dr. Duke's Phytochemical and Ethnobotanical Databases. https://phytochem.nal. usda.gov/

31. Sundarraj $S$, Ramar T, Sreevani $V$, Kaveri K, Gunasekaran $P$, Achiraman $S$, et al. $\nabla$-Sitosterol from Acacia nilotica L. induces $\mathrm{G} 2 / \mathrm{M}$ cell cycle arrest and apoptosis through c-Myc suppression in MCF-7 and A549 cells. J Ethnopharmacol. 2012;141(3):803-9.

32. Balamurugan $\mathrm{R}$, Duraipandiyan $\mathrm{V}$, Ignacimuthu $\mathrm{S}$. Antidiabetic activity of $\bigotimes$-sitosterol isolated from Lippia nodiflora $L$. in streptozotocin induced diabetic rats. European J Pharmacol. 2011;667(1-3):410-8.

33. Balamurugan $R$, Stalin A, Ignacimuthu S. Molecular docking of $\otimes$-sitosterol with some targets related to diabetes. Eur J Med Chem. 2012;47:38-43.

34. Ling WH, Jones PJH. Dietary phytosterols: A review of metabolism, benefits and side effects. Life Sci. 1995;57(3):195-206.

35. Chai JW, Kuppusamy UR, Kanthimathi MS. Beta-sitosterol induces apoptosis in MCF-7 Cells. Malaysian Journal of Biochemistry and Molecular Biology. 2008;16(2):28-30.

36. Parvez MK, Alam P, Arbab AH, Al-Dosari MS, Alhowiriny TA, Alqasoumi SI. Analysis of antioxidative and antiviral biomarkers $\bigotimes$-amyrin, $\bigotimes$-sitosterol, lupeol, ursolic acid in Guiera senegalensis leaves extract by validated HPTLC methods. Saudi Pharm J. 2018;26(5):685-93

37. Jayashree I, Geetha D, Rajeswari M. GC-MS Analysis of bioactive constituents of Glochidion ellipticum WT. Int J Pharm Sci Res. 2015;6(2546):13010-40.

38. Deora GS, Bano I. Preliminary phytochemical screening and GC-MS analysis of methanolic leaf extract of Abutilon pannosum (Forst. F.) Schlect. from Indian Thar desert. J Pharmacogn Phytochem. 2019;8(1):894-99.

39. Ogunlesi M, OkieiW, Ofor E, Osibote AE. Analysis of the essential oil from the dried leaves of Euphorbia hirta Linn (Euphorbiaceae), a potential medication for asthma. Afr J Biotechnol. 2009;8(24):7042-50.

40. Satyal P, Dosoky NS, Poudel A, Setzer WN. Essential oil constituents and their biological activities from the leaves of Cassia fistula growing in Nepal. Open Access Journal of Medicinal and Aromatic Plants. 2012;3(2):1-4.
41. Peana AT, D'Aquila PS, Panin F, Serra G, Pippia P, Moretti MDL. Antiinflammatory activity of linalool and linalyl acetate constituents of essential oils. Phytomedicine. 2002;9(8):721-6.

42. Li YC, Xian YF, Ip SP, Su ZR, Su JY, He JJ, et al. Anti-inflammatory activity of patchouli alcohol isolated from Pogostemonis Herba in animal models. Fitoterapia. 2011;82(8):1295-301.

43. Juergens UR, Dethlefsen U, Steinkamp G, Gillissen A, Repges R, Vetter $H$ Anti-inflammatory activity of 1.8-cineol (eucalyptol) in bronchial asthma: a double-blind placebo-controlled trial. Respiratory Medicine. 2003;97(3):250-6.

44. Hashim YZHY, Phirdaous A, Azura A. Screening of anticancer activity from agarwood essential oil. Pharmacognosy Res. 2014;6(3):191-4.

45. Rustan AC, Drevon CA. Fatty Acids: Structures and Properties. In: Encyclopedia of Life Sciences. John Wiley and Sons Ltd, Chichester. 2005.

46. Abdel-Aal El, Haroon AM, Mofeed J. Successive solvent extraction and GC-MS analysis for the evaluation of the phytochemical constituents of the filamentous green alga Spirogyra longata. The Egyptian Journal of Aquatic Research. 2015;41(3):233-46.

47. Pascual G, Avgustinova A, Mejetta S, Martín M, Castellanos A, Attolini CSO et al. Targeting metastasis-initiating cells through the fatty acid receptor CD36. Nature. 2017;541(7635):41-5.

48. Aparna V, Dileep KV, Mandal PK, Karthe P, Sadasivan C, Haridas M. Anti-inflammatory property of $n$-hexadecanoic acid: structural evidence and kinetic assessment. Chem Biol Drug Des. 2012;80(3):434-9.

49. Selvamangai G, Bhaskar A. GC-MS analysis of phytocomponents in the methanolic extract of Eupatorium triplinerve. Asian Pac JTrop Biomed. 2012;2(3):1329-32.

50. Agoramoorthy G, Chandrasekaran M, Venkatesalu V, Hsu MJ. Antibacterial and antifungal activities of fatty acid methyl esters of the blind-your-eye mangrove from India. Braz J Microbiol. 2007;38(4):739-42.

51. Vadivel E, Gopalakrishnan S. GC-MS analysis of some bioactive constituents of Mussaenda frondosa Linn. Int J Pharma Bio Sci. 2011;2(1):313-20.

52. Jadhav , Kalase V, Patil P. GC-MS analysis of bioactive compounds in methanolic extract of Holigarna grahamii (wight) Kurz. Int J Herb Med. 2014;2(4):35-9.

53. Al-Marzogi AH, Hameed IH, Idan SA. Analysis of bioactive chemical components of two medicinal plants (Coriandrum sativum and Melia azedarach) leaves using gas chromatography-mass spectrometry (GC-MS). Afr J Biotechnol. 2015;14(40):2812-30

54. Manorenjitha MS, Norita AK, Norhisham S, Asmawi MZ. GC-MS analysis of bioactive components of Ficus religiosa (Linn.) stem. Int J Pharm Bio Sci. 2013;4(2):99-103.

55. Fiege H, Voges HW, Hamamoto T, Umemura S, Iwata T, Miki H, et al. Phenol derivatives. In: Wiley-VCH, editor. Ullmann's Encyclopedia of Industrial Chemistry. Weinheim: Wiley VCH. 2000.

56. Kumar NR, Reddy JS, Gopikrishna G, Solomon KA. GC-MS determination of bioactive constituents of Cycas beddomei cones. Int J Pharm Bio Sci. 2012;3(3):344-50.

57. Ohno O, Ye M, Koyama T, Yazawa K, Mura E, Matsumoto H, et al. Inhibitory effects of benzyl benzoate and its derivatives on angiotensin II-induced hypertension. Bioorg Med Chem. 2008;16:7843-52.

58. Ravi S, Padmanabhan D, Mamdapur VR. Macrocyclic musk compounds Synthetic approaches to key intermediates for exaltolide, exaltone and dilactones. J Indian Inst Sci. 2013;81(3):299-312.

59. Liebert MA. Final report on the safety assessment of myristyl myristate and isopropyl myristate. J Am Coll Toxicol. 1982;1:55-80.

60. Ahmad T, Kandil F, Moustapha C. Synthesis, characterization, biological evaluation and antibacterial activity of some heterocyclic fluorene compounds derived from Schiff base. Int J Chem Tech Res. 2015;7(6):2752-62.

61. Kovács A, Vasas A, Hohmann J. Natural phenanthrenes and their biological activity. Phytochemistry. 2008;69(5):1084-110.

62. Elsharkawy E, Alshathly M, Helal M. Anti-inflammatory and chemical composition of two plants Family Asteraceae growing in Saudi Arabia. J Chem Chem Eng. 2014;8(2):157-62.

63. Karthik Prabu M, Samydurai P, Subbaiyan B, Thangapandian V. Phytochemical constituents and Gas Chromatography-Mass Spectrometry analysis of Caralluma diffusa (Wight) N. E. Br. Aerial Part. Int J Pharm Pharm Sci. 2013;4:1-4.

64. Idan SA, Al-Marzogi AH, Hameed IH. Spectral analysis and anti-bacterial activity of methanolic fruit extract of Citrullus colocynthis using gas chromatographymass spectrometry. Afr J Biotechnol. 2015;14(46):3131-58.

65. Jones TR, Calvert AH, Jackman AL, Brown SJ, Jones M, Harrap KR. A potent antitumour quinazoline inhibitor of thymidylate synthetase: synthesis, biological properties and therapeutic results in mice. European J Cancer. 1981;17(1):11-19. 


\section{GRAPHICAL ABSTRACT}

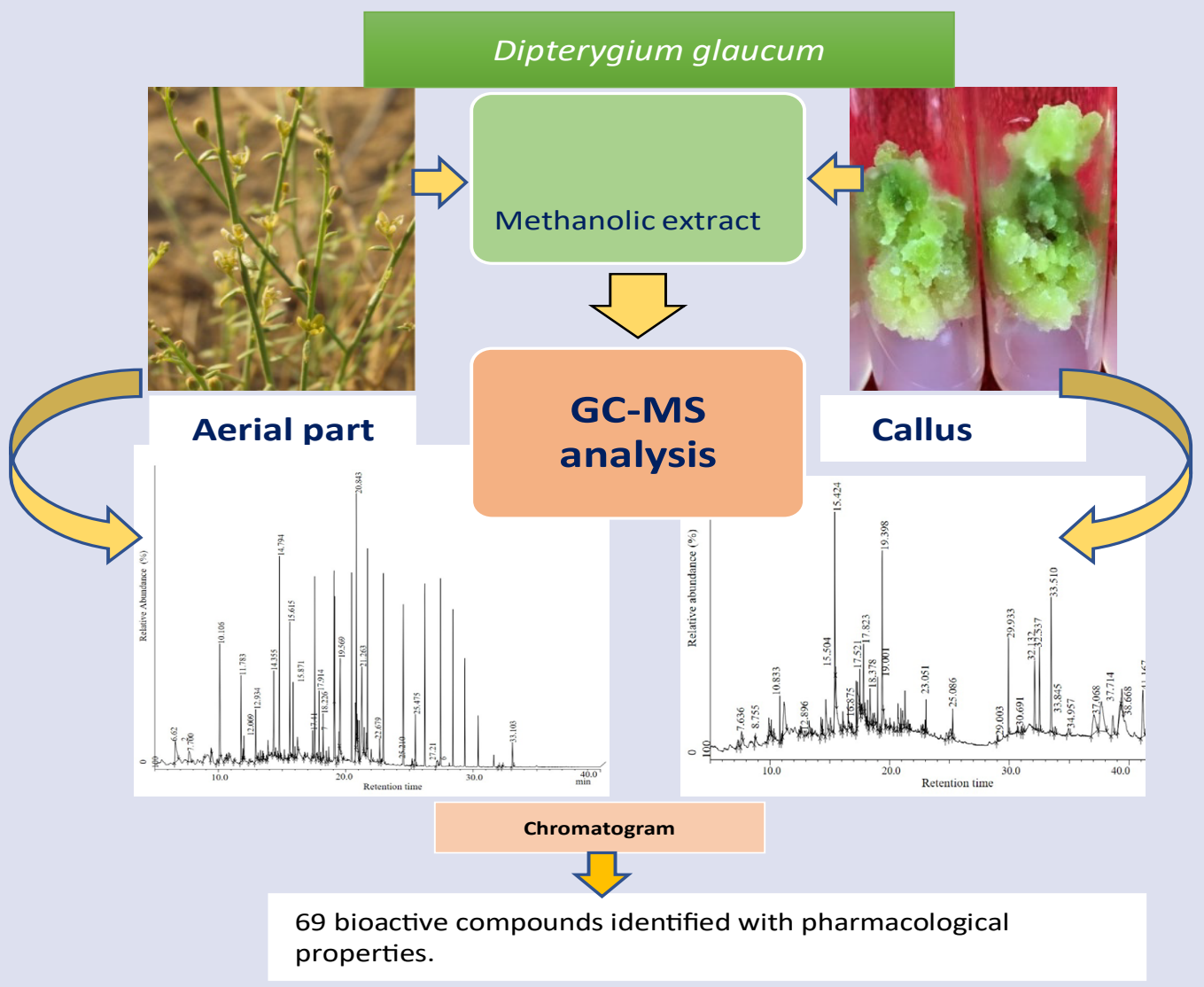

\section{ABOUT AUTHORS}

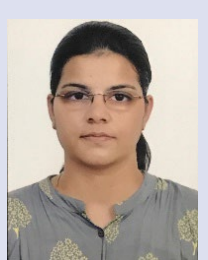

Ms. Dheeraj Choudhary is a Research Scholar and pursuing Ph.D. in Biotech Unit, Department of Botany, Jai Narain Vyas University, Jodhpur, India.

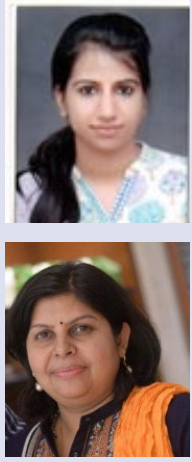

Ms. Jatan K. Shekhawat is a Research Scholar and pursuing Ph.D. in Biotech Unit, Department of Botany, Jai Narain Vyas University, Jodhpur, India.

Dr. Vinod Kataria is working as Assistant Professor at Department of Botany, Jai Narain Vyas University, Jodhpur, India. She is working in area of Plant Biotechnology and Molecular biology of Plants.

Cite this article: Choudhary D, Shekhawat JK, Kataria V. GC-MS Analysis of Bioactive Phytochemicals in Methanol Extract of Aerial Part and Callus of Dipterygium glaucum Decne. Pharmacog J. 2019;11(5):1055-63. 